\title{
Evidence Based Practice
}

\author{
Renjith George* \\ Melaka-Manipal Medical College, Malaysia
}

Submission: September 20, 2016; Published: September 20, 2016

*Corresponding author: Renjith George, Dentist, Oral Pathologist, Melaka-Manipal Medical College, Malaysia, Email: drrenjithgeorgep@gmail.com

\section{Editorial}

Evidence based practice is a new term which recently echoes in the field of health sciences. As quoted "EBP is the integration of clinical expertise, patient values, and the best research evidence into the decision making process for patient care. Clinical expertise refers to the clinician's cumulated experience, education and clinical skills. The patient brings to the encounter his or her own personal preferences and unique concerns, expectations, and values. The best research evidence is usually found in clinically relevant research that has been conducted using sound methodology [1]."

With the technological revolution, millions of results are at our fingertip through the internet. Whenever we need information or supporting evidence, majority of the health practitioners rely on internet sources. While the results are abundant in number, it is a practitioner's discretion to analyse which ones are reliable and which are not. But as it is very subjective, it is advised that $o$ have guidelines for assessing the results. Systematic reviews and met analyses help the practitioner to overcome this difficulty and to follow evidence based practice.

When the practitioner seeks information on any particular health care related condition, there are quite a few questions that arises: whom to ask, where to look for answers, how reliable are the sources, am I doing the right practice and the like. The sources of information can be text books, internet resources, experts in the field, other published literature like case reports and review articles.

But still the question stands that how reliable are they which apparently point to the quality of evidence. Here comes the relevance of clinical research. Clinical trials are time-tested reliable source of evidence to support or reject drugs or treatment methods. There are multiple trials conducted worldwide over a period of time. So, arriving at a conclusion based on multiple trials often end up in "not-conclusive" status. Emergence of systematic reviews attempts to tackle this problem. Systematic reviews and meta analyses are placed at the apex of the pyramid of clinical evidence, followed by Randomised controlled trials, cohort studies, case control studies, case series, case reports, editorials, animal research and in vitro researches at the base.

There are various organisations which promotes and conducts systematic reviews on clinical trials. Cochrane and DARE (Database of Abstracts of Reviews of Effects) are two examples to list among them. They aim to collect and extract the data from all the available data in addressing an issue and evaluate them according to the quality of evidence. As they are systematically done under specific guidelines, apparently they can be considered as the most reliable source of information. During the process of systematic review, many of he conducted researches will be excluded due to poor methodology. Eventually, the number of the sources will come down to a level which often force the authors to conclude the review stating that further research is advised due to low quality of evidence.

For these reasons, it is important to emphasise on the need of keeping up the quality of the research to follow evidence based practice in health science to deliver the best treatment for the patient.

Advances in Dentistry \& Oral Health being an upcoming journal in the field of dentistry, our authors are encouraged to consider the importance of maintaining the quality of research and evidence based practice. Clearly, our journal desires dissemination of quality evidence to ensure and better life for the patients.

\section{Reference}

1. Sackett D (2002) What is Evidence-Based Practice (EBP)? 